\title{
Tensiones heurísticas de la educación inclusiva
}

\author{
Heuristic tensions of inclusive education
}

Tensões heurísticas da educação inclusiva

\author{
Aldo Ocampo González \\ aldo.ocampo@celei.cl \\ https://orcid.org/0000-0002-6654-8269
}

Centro de Estudios Latinoamericanos de Educación Inclusiva (CELEI) ${ }^{1}$, Chile

Recibido 08 de enero 2021 | Arbitrado y aceptado 15 de febrero 2021| Publicado en 01 abril 2021

\begin{abstract}
RESUMEN
El presente artículo expone algunas de claves analíticas más significativas implicadas en la construcción del conocimiento de la educación inclusiva. El objetivo de este trabajo es analizar las trayectorias analíticas que permiten sostener que la educación inclusiva no pertenece con exactitud a la regionalización signada como educación especial. Si esta errónea vinculación ha sido establecida se debe exclusivamente a los persistentes errores de aproximación de los investigadores a su objeto y término. La inclusión no se limita a ningún campo en específico, no así, la educación inclusiva. La naturaleza del conocimiento del campo devela un conjunto de articulaciones heurísticas que inauguran una peculiar convergencia post-disciplinar. El método empleado es el de revisión documental crítica. Entre sus conclusiones, se observa que sus fuerzas definitorias asumen un carácter epistemológico neo-materialistas, una forma de escape a los sistemas logocéntricos de producción del saber.
\end{abstract}

Palabras clave: epistemología de la educación inclusiva; nomadismo; diasporismo; transposiciones; proyecto de conocimiento en resistencia

\section{ABSTRACT}

This article presents some of the most significant analytical keys involved in the construction of knowledge of inclusive education. The objective of this work is to analyze the analytical trajectories that allow us to maintain that inclusive education does not exactly belong to the regionalization designated as special education. If this erroneous link has been established, it is exclusively due to the persistent errors of approach by researchers to its object and term. Inclusion is not limited to any specific field, not inclusive education. The nature of field knowledge reveals a set of heuristic articulations that inaugurate a peculiar post-disciplinary convergence. The method used is the critical documentary review. Among its conclusions, it is observed that its defining forces assume a neo-materialist epistemological character, a form of escape from logo-centric systems of knowledge production.

Key words: epistemology of inclusive education; nomadism; diasporism; transpositions; knowledge project in resistance

\section{RESUMO}

Este artigo apresenta algumas das chaves analíticas mais significativas envolvidas na construção do conhecimento da educação inclusiva. 0 objetivo deste trabalho é analisar as trajetórias analíticas que nos permitem sustentar que a educação inclusiva não pertence exatamente à regionalização designada como educação especial. Se esse vínculo errôneo foi estabelecido, é exclusivamente devido aos erros persistentes de abordagem por parte dos pesquisadores de seu objeto e termo. A inclusão não se limita a nenhum campo específico, nem à educação inclusiva. A natureza do conhecimento de campo revela um conjunto de articulações heurísticas que inauguram uma convergência pósdisciplinar peculiar. 0 método utilizado é a revisão documental crítica. Entre suas conclusões, observa-se que suas forças definidoras assumem um caráter epistemológico neo-materialista, uma forma de fuga aos sistemas de produção de conhecimento logo-centrados.

Palavras-chave: epistemologia da educação inclusiva; nomadismo; diasporismo; transposições; projeto de conhecimento em resistência

${ }_{1}^{1}$ Primer centro de investigación creado en Chile y en América Latina y el Caribe (ALAC), dedicado al estudio teórico y metodológico de la educación inclusiva, articula su trabajo desde una perspectiva inter-, post- y para-disciplinar. Institución internacional reconocida y con estatus afiliativo por el Consejo Latinoamericano de Ciencias Sociales (CLACSO) y por el International Consortium of Critical Theory Programs (ICCTP), EE.UU 


\section{INTRODUCCIÓN}

La educación inclusiva no pertenece con exactitud a la regionalización signada como educación especial. Si esta errónea vinculación ha sido establecida se debe exclusivamente a los persistentes errores de aproximación de los investigadores a su objeto y término, así como, al gran espectro de objetos específicos que de ella se desprenden. El campo de problemas que asume este territorio ha impulsado su migración, recepción, diálogo, co-penetración, plasticidad y traducción por diversos campos que participan en su génesis y desarrollo, en tanto tópico de análisis, su fuerza sustantiva es abordada por algunos ejes de tematización de la sociología, algunos objetivos y vocabularios proporcionados por los estudios de género, luchas permanentes del feminismo, algunos ejes de investigación de la psicología, las ciencias políticas, los estudios culturales anglosajones $\mathrm{y}$, especialmente, latinoamericanos y asiáticos, el trabajo social, etc. Hasta aquí, convendría distinguir dos niveles de análisis. Un primer ámbito referido a los campos y regionalizaciones que participan del ensamblaje, germinación y organicidad del dominio y, un segundo nivel, los territorios heurístico-políticos que han capturado la fuerza del calificativo y sus giros migratorios inaugurando nuevos tránsitos, (dis)continuidades, produciendo nuevos figurales de trabajo y vocabularios para leer el presente; un presente bastante evanescente. Este giro migratorio ha establecido nuevos enlaces e incluso, ha renovado varios supuestos de trabajo, especialmente, dedicados al estudio de las formas constitutivas y regulativas de los diversos tipos de desigualdades, las identidades, las formas de exclusión, dominación y opresión que, en el terreno educativo y social, se expresan como frenos al auto-desarrollo y a la autoconstitución. Cada uno de estos enlaces funciona por co-penetración y rearticulación de sus unidades de constitución, formas heurísticas y vocabularios.

La inclusión es una teoría cuyo significante puede ser entendido en singulares direcciones. Como teoría entraña una forma que va mucho más allá de una palabra de moda, frecuentemente, utilizada por los circuitos académicos, ejes de trabajo de la política pública y de la formación de los educadores. La teoría de la educación inclusiva que propongo ofrece nuevos ángulos de visión para examinar diversas clases de problemas educativos, muchos de ellos, desconocidos o que, debido a su complejidad no logran ser comprendidos por la investigación, por ello, sostengo que este territorio se encuentra interesado en eventos raros. Trabajar sobre la emergencia de eventos raros posibilita la comprensión de temas noconocidos, es por ello que,

[...] los eventos raros son imprevistos, rompen los moldes $\mathrm{y}$ patrones habituales, y rompen la cotidianidad en cualquier acepción de la palabra. En otras palabras, los acontecimientos extremos no se explican tanto en función de la necesidad, sino de quiebres, rupturas, discontinuidades y tiempos y espacios discretos. Más exactamente, pensar en eventos raros equivale a pensar en sistemas discretos, y en absoluto en sistemas continuos (Maldonado, 2016, p.9).

La teoría de la educación inclusiva que propongo trabaja estableciendo diversas clases de aleaciones, mecanismos de conectividad y rearticulación entre una amplia variedad de perspectivas de trabajo, favoreciendo un enfoque flexible que asume la creación de un 
paradigma-otro que trabaja sobre una diversidad de problemas desconocidos $\mathrm{y}$ contingentes. Como constelación analítica articula su tarea sobre un conjunto de términos, intervenciones teóricas, premisas, definiciones de problemas y propuestas de solución que trabajan en la entridad de la justicia social y educativa, la artesanía del diseño teórico del futuro, las formas imaginativas de intervención en el presente, entre otras. Todas ellas, enmarcadas en la construcción de un nuevo figural. La educación inclusiva como teoría actualiza en un doble impulso de creatividad todos los dominios de las Ciencias de la Educación, coexiste así, un efecto paroxico y alter-epistémico devenido en un mecanismo de apertura y redoblamiento. $\mathrm{Si}$ bien, una de sus funciones es actualizar la trama constitutiva del campo y sus recursos cognitivo-metodológicos, explícita también, un efecto de apertura por redoblamiento hacia cosas inimaginadas, instaura un efecto de recuperación de las funciones sustantivas a la propia educación. El efecto alter-epistémico reside en los detalles de dicha recuperación. Trabajo para evitar que la categoría de la inclusión se vuelva sacrosanta, es intensamente contingente y política, lo que devela un estatus de complejidad. Los desarrollos institucionalizados y derivados por vía del efecto mimético imponen una comprensión fácil que la vuelve superflua.

La teoría de la educación inclusiva se convierte en un dispositivo heurístico cuando logra capturar una multiplicidad de proyectos de conocimiento en resistencias y formas metodológicas que visibilizan diversos hilos de análisis para examinar las complicidades imperceptibles con la desigualdad. Esta forma teórica construye una contra-teoría y un contra-espacio epistemológico conformado por intersecciones, traducciones y rearticulaciones de diversos recursos constructivos, inaugurando así, un ensamblaje aléctico, caracterizado por singulares mecanismos de interconexión entre diversas clases de recursos epistemológicos que juntos crean a través de la traducción, la examinación topológica y la rearticulación un nuevo figural. La construcción del conocimiento de la educación inclusiva avanza por diversas convergencias heurísticas.

\section{METODOLOGÍA}

El método empleado es el de revisión documental crítica (Flick, 1998). El foco de búsqueda estuvo centrado en investigaciones científicas, dichos trabajos fueron ubicados en bases de datos digitales y bibliográficas, tales como: Scielo, Proquest, Redalyc y Ebsco y bibliotecas electrónicas especializadas en Latinoamérica y Europa. La selección y configuración del corpus de trabajos que vertebran el presente artículo, quedó definida a partir de: a) artículos procedentes de cada uno de los principales campos de confluencia que garantizan y participan en la producción del conocimiento especializado y auténtico de la educación inclusiva, b) publicaciones que analizan la configuración de estructuras de conocimiento abiertas $\mathrm{y}$ atravesadas por signos de una apertura ambivalente $y$ rizomática y c) matrices reflexivas que abordan de manera más sofisticada las tensiones de constitución metodológicas y analíticas de territorios atravesados por múltiples convergencias heurísticas. Las fases de análisis de la información quedaron delimitadas por tres grandes momentos. La primera, caracterizada por la búsqueda y ensamblaje de un corpus de trabajos a partir de conceptos claves y de la determinación de 
rutas heurísticas ligadas a las principales influencias e intereses de producción del conocimiento de la educación inclusiva. La segunda, centrada en la emergencia de un índice de tematización coherente con cada tópico de análisis expuesto $\mathrm{y}$, finalmente, la construcción de categorías propias de análisis para vertebrar cada una de las tensiones ligadas al campo de producción epistemológico, político y metodológico de los territorios que definen la naturaleza de la educación inclusiva como proyecto de recognición de la teoría educativa contemporánea.

\section{DESARROLLO Y DISCUSIÓN}

\section{¿Qué es exactamente la educación inclusiva?: tensiones en su teoría y metodología}

Tal como he sostenido en diversos trabajos, una de las dificultades permanentes que enfrenta este dominio describe un índice de singularidad -identidad del campo- de baja intensidad, para ser más preciso, adopta un estatus de desconocimiento. Si bien, muchos investigadores dedicados al género creen saber o conocer la inclusión, al momento que deben explicarla no saben definirla o abordarla en profundidad. El debate acerca de cómo conceptualizar y utilizar la inclusión y la educación inclusiva no es el todo claro; es esta ambigüedad e ininteligibilidad lo que, en cierta medida, la hace atractiva. Sin embargo, es necesario avanzar y comprender cabalmente su índice de singularidad, ambos son conceptos que estimulan la imaginación, la lucha sistemática por la creación de otros mundos y modos de relacionamiento $\mathrm{y}$ coordenadas de alteridad. Sus tensiones, presiones y obstrucciones metodológicas quedan definidas por el carácter abierto y en permanente movimiento y devenir, es lo que inscribe sus desarrollos metodológicos en términos de dificultad creciente. Lo abierto al igual que la interseccionalidad dificultad que los contornos del campo sean delimitados en la centralidad de una única forma metodológica. Esto enfrenta otro problema definitorio: no explica con exactitud cómo estudiar su campo de fenómenos heurísticos, metodológicos, conceptuales, visuales, político-éticos, etc. La inclusión y la educación inclusiva reafirman un estatus de metáfora, una explicación creada por diversos grupos y empleada con diversos propósitos y fines. Si bien, su surgimiento no está claro, así como, sus usos heurísticos y metodológicos, lo cierto es que, estas, han surgido entremedio de una gran variedad de espacios discursivos, proyectos de conocimiento y debates, explicitando tres grandes compromisos: a) debates discursivos y ético-políticos sobre el alcance y contenido de la inclusión y la educación inclusiva, ambas categorías demuestran un estado hipónimo, como proyecto teórico y metodológico, b) intervenciones políticas con lente inclusivo y c) prácticas de investigación con un marco imaginativo para fundamentar el diseño teórico y ético del futuro.

¿Cuáles son las dificultades para traducir la teoría y epistemología de la educación inclusiva en metodología? Un aspecto medular que a su vez, es una afirmación, explícita que todos los sujetos atravesados por el significante de lo inclusivo son afectados por diversos ejes de desigualdad. Asume además, "que la subjetividad es constituida por vectores que se refuerzan mutuamente la raza, el género, la clase y la sexualidad" (Nash, 2008, p.2). Hoy la educación inclusiva y la inclusión constituyen temas contingentes y de interés creciente por diversos campos ligados al 
desarrollo humano, pero, en menor grado constituye un enfoque popular en la interioridad de la investigación social. Este se ha vuelto más visible en los últimos años, siempre desde un efecto restrictivo y normativo. Como herramienta analítica es una de las principales y más poderosas para avanzar en la creación de otros mundos, es una poderosa forma imaginativa y una teoría que se aplica a todo y a todos. Lo cierto que es la inclusión se bate en sus contornos definitorios entre quienes defienden una posición a favor de las perspectivas asimilacionistas, otros que trabajan para correr el marco a favor de una teoría de las identidades marginadas o bien, una teoría general de recambio de la educación. La educación inclusiva y la inclusión son, cada una a su forma, conceptos teóricos relevantes para pensar los grandes temas del mundo actual.

La pregunta por la teoría es clave en la organicidad de un proyecto de investigación, no solo define el contenido ideológico y crítico, sino también, su dimensión política. En este sentido, la teoría de la educación inclusiva que propongo se co-penetra con lo político. Razón por la cual, será fundamental describir el tipo de legitimidad académica que produce e instala, así como, el valor social que materializa en la diversalidad de estructuras de los sistemas-mundo. Para Dalmaroni (2020) "un cierto estado de la teoría en un sentido amplio -digamos, un estado del pensar críticopermite advertir y construir ciertos problemas y no otros" (p.34). La inclusión inaugura una nueva modalidad de intervención en los modos de pensar y abordar los fenómenos que convergen en sus territorios, se convierte en una estructura estructurante del repertorio de problemas constitutivos del mundo actual.

Si la educación inclusiva designa un tipo de teoría viajera, mutante, nómada y con una morfología de funcionamiento con proximidad a la metáfora del virus ${ }^{1}$ descrita por Deleuze y Guattari (1970), entonces, sostendré que su función ya no actúa en términos de un vector que impone y sanciona determinados tipos de verdades sobre discusiones disciplinares particulares y sus paradigmas, es algo que dice mucho más que esto, actúa como un sistema de orientación, supera el interés de las discusiones propias de determinados campos disciplinares producto que su objeto desborda los paradigmas rectores de cada una de ellas. Pero, si la teoría de la educación inclusiva entraña una operación post-disciplinar, entonces, ¿de qué se desplaza? o ¿desde dónde? Emerge así, una nueva advertencia: lo post- en este campo no asume las demandas explícitas de su significante, más bien, opera en proximidad a la ruptura, a la transitividad nolineal, a la emergencia de otras imágenes de pensamiento aun no-vislumbradas. Lo postopera en sintonía con la fractura de los modos instituidos del saber. Examinemos a continuación, aunque brevemente, los usos del vocablo teoría.

Tal como comenta Biset (2020) la teoría puede ser significada como la aventura de la mirada, aquello que actúa por excedencia disciplinar, involucra una modalidad de recognición en la aproximación e interacción con diversas clases de objetos. La teoría de la educación inclusiva que propongo plantea una nueva forma de indagar la especificidad de sus objetos visuales, teóricos, analíticos, políticos, etc.; construye, como tal, un "nuevo materialismo o ciertos modos de pensar la vida" (Biset, 2020, p.2). En este sentido, el significante de teoría al que adhiero no apela exclusivamente a la ruptura de las disciplinas,

1 Metáfora que alude a singulares formas de contagio, mutación y diseminación de ideas. 
más bien, busca la reinvención de estas, trazando nuevas coordenadas heurísticas que surgen del encuentro rearticulador de variados continentes que se asumen como movimientos teóricos centrales en el desprendimiento de sus herencias o cadenas heurísticas bidireccionales que inauguran una mimesis normativa. Cada uno de los campos de confluencia y continentes concursantes en la emergencia de su rostricidad auténtica, actúan "como indicios de un gesto teórico que permiten un punto de partida" (Biset, 2020, p.3) alterativo.

La pregunta por la educación inclusiva y la inclusión en tanto metodología inscribe su análisis en los niveles de articulación de ambos fenómenos en las estructuras constitutivas/regulativas del sistema-mundo. Ambas categorías designan un poderoso lazo que rearticula la fuerza de lo estructural y lo micropráctico; dos de las principales características de este fenómeno. Une ambas fuerzas de relación creando nuevos diagramas de relacionamientos, particularmente, "nuevos diagramas contingentes de posiciones $y$ situaciones relacionales y transitivas" (Richard, 2020, p.s/r.) que fomentan otras coordenadas de alteridad para acoger a la multiplicidad de singularidades, espacialidad que busca dar respuesta a través de la metáfora de 'vector proyectivo' de identidades $\mathrm{y}$ pasiones fracturadas, disconformes $\mathrm{o}$ devaluadas por las diversas capaz de la sociedad. Al concebir la educación inclusiva como metodología discute y profundiza en el tipo y naturaleza de operación en el que esta se inscribe. Al respecto, cabe recuperar la afirmación de Ocampo (2020) sobre el nivel de operación de la inclusión, cuya arquitectura acontece de forma multinivel y trasrelacional, conectando lo estructural -afecta y trasforma a las reglas de funcionamiento institucional de la sociedad, las que son convertidas en reglas de funcionamiento de los sistemas educativos- y la red de acontecimientos micro-escalares o microprácticos que organizan la red de interacciones cotidianas, espacialidad que incorpora la trama fenomenológica y sus objetos de orientación en el pensamiento.

La inclusión como metodología opera estableciendo lazos conectivos y anudamientos críticos entre las fuerzas de desarrollo estructural y micropráctico, crea un nuevo diagrama de posiciones, relacionamientos $\mathrm{y}$ coordenadas de alteridad. La pregunta por los niveles de operación comparte con la interseccionalidad los cuatro niveles del análisis multinivel expuestos por Anthias (1998): a) experiencias, b) prácticas intersubjetivas, c) regímenes institucionales y d) sistemas simbólicos y discursivos. Cada una de estas dimensiones sugieren "aprender a "hacer" un análisis que incluye varios niveles" (Davis, 2014, citado en Luzt, 2015, p.40). La inclusión es el principal pilar teórico de la investigación educativa, se encuentra en todas partes, devela una compleja trama fenoménica, está constituida por diversas singularidades ontología materialista- y relacionada con diversas clases de diferencias. Esto hace que la inclusión no sea tratada como una categoría totalmente independiente.

Un dilema definitorio particular que enfrenta la trama investigativa de la educación inclusiva consiste en crear mecanismos para subvertir la ficción objetivista de la inclusión y, consiguientemente, crear condiciones de justicia para que determinados grupos atravesados por el significante de la subalternidad y la ausencia de reciprocidad social y ético-política, evitando que dichas formas de justicialidad epistémica devengan en prácticas de representación sociopolítica. 
Emerge aquí, una interrogante central: ¿cómo dar cuenta del papel del investigador en la interioridad de este dominio? La inclusión asume entre sus múltiples objetivos de trabajo la comprensión de la mecánica interna de formas específicas de opresión experimentadas por diversas personas, fenómeno relacional, no categorial; supera de esta forma, los marcos rígidos instituidos por la gramática del binarismo y del individualismo-esencialismo, espacialidad analítica en el que los términos operan bajo relaciones mutuamente excluyentes. El estudio de las formas de desigualdad reclama intensamente la superación de este pseudomarco de aproximación a su mecánica constitutiva. La regulación de las estructuras del sistema-mundo se encuentra atravesada por diversas formas de opresión y dominación, cada una de estas, concebidas como frenos al auto-desarrollo y a la auto-constitución.

La naturaleza de la educación inclusiva ratifica un carácter interseccional producto de encontrarse atravesado por diversos ejes de constitución y enredos genealógicos, es algo que va configurándose en diversas capas y convergencias epistémicas, metodológicas y categoriales; devela un interés teórico y político, intensamente conectado con las demandas del feminismo, la interseccionalidad, la filosofía de la diferencia, los estudios de la mujer, los estudios postcoloniales, los estudios queer, los estudios de género, los estudios de la subalternidad, entre otros. Cada uno de estos enfoques al confluir en el ensamblaje del campo mediante complejas formas de traducción -algo que se traslada de un lugar a otro, muchos de sus recursos se contaminan, se pierden o bien, experimentan un cambio transformador que se articula en términos de "movimiento y renovación de significados" (Richard, 2019, p.s/r) y rearticulación -giro creativo- para colocar en práctica cada uno de estos supuestos teórico-metodológicos. Cuando hablo de epistemología de la educación inclusiva lo hago para explorar lo que significa conducir la investigación en este campo, sus formas de representación de los hallazgos y sus modalidades de comunicación de estos ante la comunidad científica. ¿Cómo utilizar la epistemología de la educación inclusiva como enfoque de investigación y herramienta de intervención en los sistemas-mundo?, ¿qué implica hacer este tipo de investigación y qué implica hacerlo?, ¿cómo pensar a través de este enfoque? Si atendemos a la especificidad de cada una de estas interrogantes sostendré que no existe una visión homogénea de inclusión, tal como reafirma la experiencia mimética de lo especial, sino que, existen múltiples formas de practicar y entender su función y alcance. Esta afirmación confirma lo que he sostenido en diversos trabajos, que no existe una única forma de inclusión y de educación inclusiva, no hay una respuesta estable ni correcta, sino que, un crisol de posibilidades y horizontes de sentido.

Una de las características generales de la investigación en educación inclusiva consiste en superar los vicios y remanentes del apartheid de los dominios teóricos que configuran proyectos de conocimiento y alianzas imperceptibles de la investigación con la desigualdad. Otra de sus manifestaciones centrales consiste en descentrar el conocimiento subyugado que permite recomponer y transformar la experiencia (inter)subjetiva de múltiples colectivos de ciudadanos. Se interroga permanentemente acerca del corpus de conocimientos, categorías, objetos visuales y metodologías de investigación que quedan relegadas o atravesadas por mecanismos de violencia 
epistémica situándolo por fuera del discurso dominante o en su defecto, en posiciones de desigualdad. Esta empresa es aquella que organiza la trama mono-céntrica y nomocéntrica que funda el régimen especialcéntrico para justificar indebidamente la rostricidad y susurro de lo inclusivo. La inclusión tiene por objeto alterar las estructuras sociales, políticas y culturales de opresión, el mismo propósito asume en un plano estructural y micro-escalar la educación inclusiva, es decir, alterar las formas opresivas que se imbrican y afectan al funcionamiento de las estructuras de escolarización su dimensión formal e informal responsables de la producción de poblaciones en riesgo o ubicados en las fronteras del derecho 'en' la educación. Nos encontramos en presencia de un problema de representación del conocimiento disponibilizado y significado como parte del gran campo de objetos visuales, teóricos, analíticos, metodológicos, éticos y políticos, etc. de lo inclusivo. Un aspecto espinoso consiste en develar el tipo de estructuras de poder a las que se enfrenta y le interesan a la inclusión y la educación inclusiva. El régimen especial-céntrico nos conduce al sesgo proporcionado por el individualismo-metodológico; este es un régimen que se encuentra en el interior de dicha gramática.

¿Qué tipo de conocimiento produce la educación inclusiva para combatir cada una de las formas de desigualdad, vulnerabilidad, precariedad de la vida material y subjetiva, entre otras?, ¿quién puede producir este conocimiento?, ¿cuáles son sus derivativas metodológicas? Las formas de investigar y producir el conocimiento en este singular, contingente y complejo contexto asumen que no siempre su conocimiento puede obtener y validarse mediante dispositivos de objetividad heurística. Sin duda, esta forma crearía un mecanismo de dependencia epistemológica de carácter normativa. La especificidad del campo se sostiene, en parte, en un mecanismo epistemológico situado y posicionado. No obstante, el conocimiento especializado de la educación inclusiva se construye, singularmente, mediante complejas formas de diasporismo y nomadismo epistemológico colocando en jaque diversas clases de razonamientos y reclamos de orden universalistas. Si la objetividad positivista es un factor estéril en la investigación de lo inclusivo, en superación de ello, propongo un sistema de objetividad inclusivo, una forma diferente de entender los marcos de objetividad del conocimiento de este territorio; es un criterio en movimiento y en dislocación permanente, coincide en determinados puntos con la propuesta de Harding (1993) procedentes desde el feminismo. Es un saber que no solo es aplicable a un determinado contexto, debido a sus características de ambivalencia, no es posible hablar de una única y estricta forma de inclusión. Son diversas formas de inclusión, una constelación. Nos enfrentamos a la búsqueda de nuevos criterios de validación del conocimiento educativo.

La pregunta por los criterios de validación del conocimiento especializado de la educación inclusiva nos remite al estudio y examinación de las coordenadas de educacionismo e interacción con determinados marcos de valores y formas culturales, en su mayoría, de la zona imaginaria del ser, lo que hace que la profundidad de los problemas reales y contingentes de la desigualdad, la opresión y la dominación no sean del todo conscientes de tales problemáticas. Para ello, la inclusión debe ayudar a liberar la zona del no-ser. La relación de muchos investigadores con el corpus de 
experiencias multiposicionales de sujetos inscritos en la zona del no-ser influye fuertemente en su consciencia de relación y sus compromisos políticos devienen en la creación de nuevas teorías de formas inconscientes que contribuyen a asumir formas precarias de intervención en algunos de los problemas medulares del presente. En este punto, observo necesario recuperar la noción propuesta en 1984 por Rich, denominada: política de ubicación respecto del fenómeno investigado, el tipo de compromisos $\mathrm{y}$ las conexiones con otras posiciones que ayudan a inscribirse dentro de cada investigación. Ciertamente, los investigadores dedicados al género de la educación inclusiva reafirman múltiples posiciones respecto del gran abanico de fenómenos que conforman su dominio. Cada investigador posee una ubicación diferenciada atravesada fuertemente por sus esquemas de socialización, subjetividad y otras formas de historicidad de su biografía y consciencia. La sección del sintagma referida a 'ubicaciones' según van Witteloostuijn (2017) "no solo se refiere al sentido literal de la palabra, es decir, la ubicación geográfica, sino también la ubicación histórica y otros marcadores de su identidad. Ella habla de sus lugares como su género, su raza, su religión, la situación política en la que se encuentra" (p.8). La política de ubicación devela que el campo de la inclusión no es un movimiento homogéneo. Las ideas y los sistemas de razonamientos no son fijos y se encuentran en permanente movimiento.

El conocimiento siempre se encuentra inscrito en una singular posición social y formación social específica, la posición que asume cada investigador es responsable de producir conocimientos diferentes. Cada fenómeno investigado se encuentra atravesado por diversos lugares de ubicación y relacionamiento con cada fenómeno interactuante. Es un campo de múltiples dispositivos de focalización de cada fenómeno. Política de ubicación del investigador y teoría del punto de vista, dos conceptos que el campo investigativo de la educación inclusiva toma prestado del feminismo sometiéndolos a traducción, desempeñan un papel crucial en la construcción de sus contornos metodológicos. La experiencia es una de las principales formas metodológicas de indagación; complementa Haraway (1988) "estoy abogando por la política y epistemologías de ubicación, posicionamiento y situación, donde la parcialidad y no la universalidad es la condición de ser escuchado para hacer afirmaciones de conocimiento racional" (p.589). La educación inclusiva entraña la tarea de restaurar el significado del mundo que habitamos.

\section{DISCUSIÓN}

\section{La inclusión como fenómeno es dinámica, cambiante y relacional. Construye una singular constelación analítico-metodológica}

Una de las principales características epistemológicas de la educación inclusiva es el cambio y el movimiento, premisas cruciales en la configuración de las denominadas epistemologías de la dispersión. Para que la inclusión y su conocimiento -proyecto teórico en resistencia y apelación pragmática de carácter micropolítica- consoliden nuevas formas de justicia educativa debe ser cambiante y operar en lo que Zemelman (1991) denomina bajo la metáfora de 'algodado-dándose-por-dar'. El cambio es una forma de resistir ante la mutación de las condiciones sociales. 
El campo de fenómenos de la educación inclusiva al expresar mayoritariamente un carácter extra-teórico, manifiesta que estas se encuentran en permanente movimiento formando un saber inacabado, en devenir y abierto hacia presentes y futuros inciertos y desconocidos, otorgando una gran dosis de imaginación y creatividad. El gran campo de fenómenos visuales, analíticos, metodológicos, éticos y políticos que conforman la circunscripción intelectual denominada educación inclusiva enfrenta el desafío de "explicar estas relaciones variables y en desarrollar análisis sobre cómo estos puntos en común se experimentan de manera diferente" (Hill Collins, 2008, p.130). La educación inclusiva es un diálogo con diversos proyectos de justicia social, clave en la comprensión de la singular constelación analítica que este campo designa. El movimiento permanente es una de las principales características heurísticas del campo, es esto lo que hace que el conocimiento que construye forje un saber del presente. La pregunta por el movimiento implica indagar acerca de los lugares por donde ha transitado, aterrizado y viajado, así como, el tipo de itinerarios que expresa. Sin embargo, no deja en claro qué es lo que esta hace. Aquí la interrogante transita hacia lo que hace la inclusión en el mundo y, particularmente, lo que hacen sus agentes para comprender profundamente lo que esta teoría puede hacer. Sin duda, este punto sigue aún en un plano de oscuridad y turbiedad, producto de la ausencia de esquemas críticos para estudiar los signos del presente.

La inclusión se convierte en una forma de navegación teórica que fomenta la exploración de formas creativas para comprometerse afirmativamente con el presente, reconoce que las nociones de 'inclusión' y 'educación inclusiva' develan una naturaleza poliédrica, elástica y nomadista. En su vinculación con el estudio de las desigualdades e injusticias varias concebidas en términos de opresiones y dominaciones, brinda un marco analítico que va más allá de la mera superposición de ejes diferenciales, sino que, consolida una analítica y una praxis crítica de carácter multinivel y dinámica, destinada a superar las teorías sociales existentes con el objeto de profundizar el análisis de las desigualdades. La inclusión es un concepto y un significante popular en diversos campos de estudio y movimientos, en su configuración convergen diversas tradiciones filosóficas, alberga una amplia variedad de cuestiones políticas y tensiones teóricas. El dominio de la educación inclusiva es un campo ensamblado por rearticulación de múltiples prácticas teórico-metodológicas heterogéneas que tensionan las formas liberales en las que han sido inscritas las funciones de dicho dominio.

La inclusión en tanto campo y fenómeno no solo se moviliza entre diversas clases de recursos constructivos y ensamblajes heurísticos, sino también, a través de sujetos cambiantes -concepción ontológica materialista-, sus movimientos reflejan un prisma que conecta, rearticula, media, disloca y gira una serie de métodos, metodologías, territorios de análisis, compromisos éticos, proyectos políticos, campos y sub-campos académicos, discursos, conceptos, sujetos, etc., esta singular malla de conexión reticular por redoblamiento adhiere una concepción más amplia de movimiento, fomentando la comunicación entre cada uno de estos recursos constructivos que inauguran un singular dominio aléctico. Buena parte del aprendizaje de la educación inclusiva se construye a partir de patrones localizados de pensamiento, 
acciones que acontecen en la micropraxis, es decir, espacio de regulación y encarnación fenomenológica del conjunto de interacciones propias de la cotidianidad. La educación inclusiva constituye "una intervención contrahegemónica y transformadora en la producción de conocimiento, el activismo, la pedagogía y las coaliciones no opresivas" (Cabardo, Crenshaw y Tomlinsom, 2013, p. s/r.). La educación inclusiva como campo intelectual genera diversas clases de compromisos con la teoría, esto se encuentra determinado por las diversas posiciones de cada uno de los recursos constructivos que participan de su ensamblaje.

Si bien los términos inclusión y educación inclusiva expresan un estatus hipónimo, es decir, uno contiene al otro, trazan un itinerario de interdependencia; cada una a su forma, han formado un complejo compromiso epistemológico atravesado por diversas convergencias heurísticas que van desde lo interdisciplinario a lo extra-disciplinario, transitando hacia acciones de carácter transdisciplinario para llegar a consolidar una singular base de carácter post-disciplinar. A su vez, traza un peculiar compromiso global de afectación a todos los campos del desarrollo humano, es un fenómeno que se mueve a través del tiempo y de singulares instrumentos conceptuales develando agenciamientos epistémicos los que son rearticulados y actúan por redoblamiento permanente y traducción para dar vida al campo discursivo de lo inclusivo. La educación inclusiva se moviliza y trabaja sobre diversos problemas de carácter contingentes, no en disciplinas como funcionan las epistemologías normativas. En efecto, es urgente avanzar en los dilemas definitorios del campo y, muy especialmente, en la caracterización de "los debates sobre su alcance y capacidad teórica, marca algunos de sus viajes disciplinarios y globales y explora la trayectoria futura de la teoría" (Cabardo, Crenshaw y Tomlinsom, 2013, p. s/r.). La premisa del movimiento puede ser empleada de diversas formas -concepto maleta-, una de ellas nos invita a pensar acerca de cómo se ha movilizado la inclusión y hacia donde se están dirigiendo sus efectos.

\section{CONCLUSIONES}

Los bordes críticos del término la describen como un objeto y campo de investigación, como un dispositivo heurístico y una estrategia analítica y una praxis crítica; tanto la inclusión como la educación inclusiva se encuentran enraizadas en el aporte de diversos enfoques, perspectivas y teorías, tales como, los estudios de género, los estudios post-coloniales, los estudios de la subalternidad, los estudios culturales, los estudios de la mujer, los estudios queer, los estudios visuales, el feminismo, la interseccionalidad, la filosofía analítica, política y de la diferencia, el antirracismo, los estudios críticos de sociología, entre otras. La confluencia disarmónica de cada uno de estos campos forma un territorio aléctico, nomadista y diaspórico. Una advertencia analítica antes de avanzar: cuando la retórica visual, la gramática conceptual y la fuerza analítica de lo inclusivo se travestiza con lo especial, funciona produciendo y legitimando la marginación. Esta forma de inclusión -régimen neo-especial o normo-céntrico- devela cómo determinados discursos institucionalizados legitiman relaciones de poder. Sin embargo, la caracterización del diario de viaje del campo sigue siendo uno de sus principales enigmas.

La teoría de la educación inclusiva sostiene que esta nunca se encuentra fija, ni 
tampoco se hace ni agota por sus articulaciones, sino que es algo siempre en movimiento, en curso y en devenir. Una teoría en movimiento asume que "existe potencialmente siempre otro conjunto de preocupaciones a las que se puede dirigir la teoría, otros lugares a los que se puede trasladar la teoría y otras estructuras de poder que se pueden desplegar para examinar" (Cabardo, Crenshaw y Tomlinsom, 2013, p. $\mathrm{s} / \mathrm{r}$.). La producción del conocimiento de la educación inclusiva a través del movimiento da cuenta de un saber en permanente apertura y giro, lo que le asigna un estatus provisional e inacabado en el abordaje de determinados femémonos, es un campo de trabajo en progreso es esto lo que traza la posibilidad de llevar a otros contextos de análisis y de trabajo la inclusión. Si bien, sus territorios enfrentan la tarea crítica de redescubrir y recomponer su índice de singularidad -identidad científica del campo-, empresa que solo será posible mediante un doble impulso de creatividad, demuestra la fertilidad de pensar su red objetual en términos de entidad contenida o bien intentar comprender el término, campo y fenómeno como estructura-agente repleto de intereses y tareas específicas, lo que hasta cierto punto puede ayudar a clarificar sus contornos.

\section{REFERENCIAS}

Anthias, F. (1998). Rethinking Social Divisions: Some Notes Towards a Theoretical Framework. Sociological Review, 46(3), 505-535

Biset, E. (2020). Escena postextual de la teoría. Recuperado el 21 de noviembre de 2020 de:

https://www.academia.edu/44492785/Es cena_postextual_de_la_teor\%C3\%ADa

Cabardo, D., Crenshaw, K. \& Tomlinsom, B.,
(2013). INTERSECTIONALITY: Mapping the Movements of a Theory. Recuperado el 13 de diciembre de 2020 de: https://pubmed.ncbi.nlm.nih.gov/252851 50/

Davis, K. (2014). Intersectionality as a Xritical Mathodology. In Lykke, N. (Ed.) Writing Academic Texts Differently: Intersectional Feminist Methodologies and the Playful Art of Writing (pp. 268-281). New York: Routledge

Dalmaroni, M. (2020). La investigación literaria. Problemas iniciales de una práctica. La Plata: Ediciones UNLP

Deleuze, G. \& Guattari, F. (1970). La lógica de sentido. Barcelona: Edit. Six Barral

Flick, U. (1998). Introducción a la Investigación Cualitativa. Madrid: Morata

Haraway, D. (1988). Situated Knowledges: The Science Question in Feminism and the Privilege of partial perspective. Feminist Studies, 14(3), 575-599

Hill Collins, P. (2008). "Rasgos distintivos del pensamiento feminista negro", en: Fabardo, M. (Edit.). Feminismos negros. Una antología. (pp.99-133). Madrid: Traficantes de Sueños

Maldonado, C. (2016). El evento raro. Epistemología y complejidad. Recuperado el 04 de julio de 2020 de: https://www.moebio.uchile.cl/56/maldon ado.html

Nash, J.C. (2008). Re-thinking intersectionality. Feminist Review, 89(1), 1-15

Ocampo, A. (2020). En torno al verbo incluir: performatividades heurísticas de la educación inclusiva. Quaest.disput, 13 (27), 18-54

Oppermann, S. (2010). The rhizomatic trajectory of ecocriticism. Recuperado el 27 de noviembre de 2020 de: https://ecozona.eu/article/view/314

Richard, N. (2019). Undoing and Redoing gender: theory, criticism and politics. Recuperado el 02 de enero de 2021 de: https://palabrapublica.uchile.cl/2019/05/ 14/undoing-and-redoing-gender-theorycriticism-and-politics/ 
Richard, N. (2020). De la revuelta social a la nueva constitución en Chile. Conferencia leída el 16 de diciembre de 2020 en el Centro Cultural Kirchner. Recuperado el día 28 de diciembre de 2020 de: https://www.youtube.com/watch?v=pIl8S JEW9Hw

Van Witteloostuijn, A. (2017). Applying intersectionality as a method a critical analysis of how to apply feminist and intersectional methodologies in qualitative research. Recuperado el día 03 de diciembre de 2020 de: https://dspace.library.uu.nl/bitstream/ha ndle/1874/365488/Thesis\%20Gender\%2 0Studies\%20-

\%20Amber\%200ttilie\%20van\%20Wittel oostuijn\%20\%283841359\%29\%20-

Zemelman, H. (1991). Los horizontes de la razón. Dialéctica y apropiación del presente (2 vols.). México: Anthropos 